\title{
Experiments on a Gas Gun for Target Injection in Inertial Fusion Energy
}

\author{
Soichiro SAKAE, Hiroyuki HAYASHI, Tomoaki KITABATAKE, Takuya MATSUMURA, \\ Takuma ENDO and Takayoshi NORIMATSU ${ }^{1)}$ \\ Department of Mechanical Engineering, Hiroshima University, Higashi-Hiroshima 739-8527, Japan \\ ${ }^{1)}$ Institute of Laser Engineering, Osaka University, Suita 565-0871, Japan
}

(Received 30 November 2008 / Accepted 30 April 2009)

\begin{abstract}
Projectile-shooting experiments using a simple gas gun were carried out to address issues in target injection technology for fast-ignition-type inertial fusion energy. The gas gun consisted of a high-pressure gas reservoir, quick solenoid valve, and smooth-bore acceleration tube. Simple cylindrical projectiles were accelerated at room temperature by nitrogen gas and shot into a diagnostic chamber. In these experiments, we measured the flight speed, direction, and attitude of projectiles of different lengths. Longer projectiles showed better flight performance.
\end{abstract}

(c) 2009 The Japan Society of Plasma Science and Nuclear Fusion Research

Keywords: inertial fusion energy, target injection, gas gun, smooth bore, cylindrical projectile

DOI: $10.1585 /$ pfr.4.S1006

In fast-ignition-type inertial fusion energy (IFE), a target that is a spherical fuel capsule with a cone [1] is injected in the center of a reaction chamber and irradiated by laser beams for compression and heating (ignition). Therefore, a target injector is required to inject a target with a specified attitude, speed, and direction. The required flight speed and direction are approximately $50-400 \mathrm{~m} / \mathrm{s}$ and one mrad respectively, strongly depending on the design of the IFE power plant and progress of other key technologies such as laser-beam steering [2-4]. The required flight attitude is determined by the shape of the cone guiding the laser beam for ignition. Typically, the axis of the cone should be directed to the ignition beam within two degrees.

A gas gun is a promising candidate for a target accelerator, and a prototype of a gas-gun-based target injection system was designed and tested with emphasis on the flight speed and direction [2-4]. In this study, we report the results of projectile-shooting experiments using a simple gas gun, focusing on flight attitude.

The experimental arrangement is schematically shown in Fig. 1. The gas gun is composed of three parts: a gas reservoir, solenoid three-way valve, and acceleration tube. The gas reservoir has a volume of $0.017 \mathrm{~m}^{3}$, which is approximately 100 times as large as that of the acceleration tube. The solenoid three-way valve is used to control projectile shooting. The acceleration tube has a smooth bore. A projectile is shot into a diagnostic vacuum chamber. In the present experiments, the initial pressure of the gas reservoir was $300 \mathrm{kPa}$ and that of the diagnostic chamber was $300 \mathrm{~Pa}$.

The length of the acceleration tube is $2193 \mathrm{~mm}$ and

author'se-mail: takumaendo@hiroshima-u.ac.jp the inner diameter is $10.21 \mathrm{~mm}$, as shown in Fig. 1. Simple cylindrical projectiles, which represent sabots protecting fuel capsules during acceleration in a future target injector, were used. Figure 2 shows the projectiles we used. They were made of DURACON® acetal copolymer (a kind of polyoxymethylene resin; Polyplastics Co., Ltd., Tokyo). Their diameter was $10.15 \mathrm{~mm}$. The lengths of the projectiles were $10.5,21.0,42.0$, and $84.0 \mathrm{~mm}$. The mass of the 10.5 -mm-long projectile was $1.19 \pm 0.02 \mathrm{~g}$. The masses of other projectiles were proportional to their lengths.

The flight speed was measured by a laser-path-cut method. A flying projectile cut a laser beam path twice during its flight, and the timings of the cuts were observed using a photodiode and an oscilloscope. The flight direction was measured by using a target catcher at the end of the diagnostic chamber. We carefully inspected the position at which the projectile was stopped. The flight attitude was directly observed using a series of LED flashes and a digital still camera. The shutter of the still camera was opened for sufficient time. The timing, pulse duration, and number of LED flashes were controlled using a highspeed pulse generator triggered by the laser signal used for speed measurement.

The duration during which the valve was opened was determined so that the disturbance by the accelerating gas was minimized without lowering injection speed. The normalized valve-open duration $\tilde{\tau}$ was defined by

$$
\tilde{\tau}=\frac{\Delta t_{\mathrm{valve}}}{\Delta t_{\mathrm{acc}}}
$$

where $\Delta t_{\text {valve }}$ and $\Delta t_{\text {acc }}$ are the valve-open duration and projectile-acceleration duration respectively. The projectile-acceleration duration $\Delta t_{\text {acc }}$ was evaluated by the 


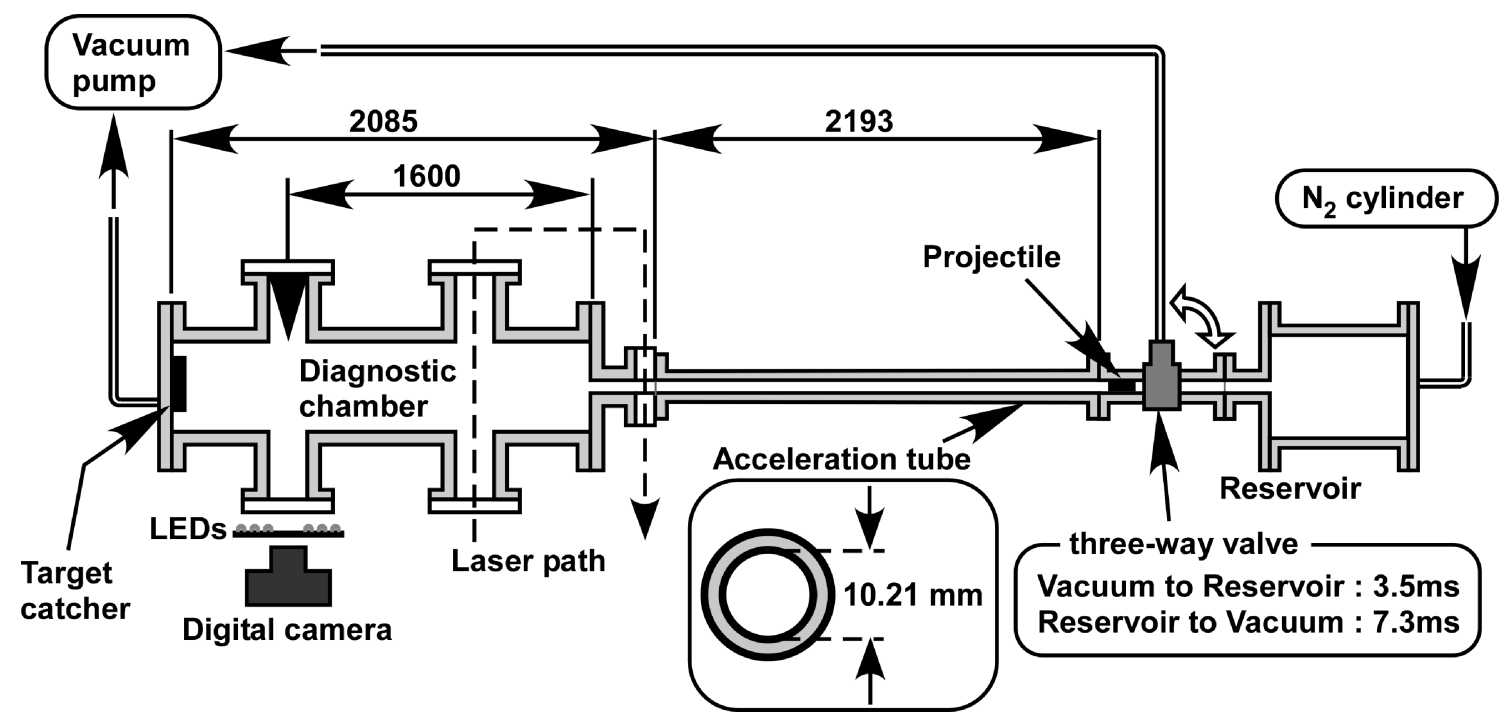

Fig. 1 Schematic of experimental arrangement.
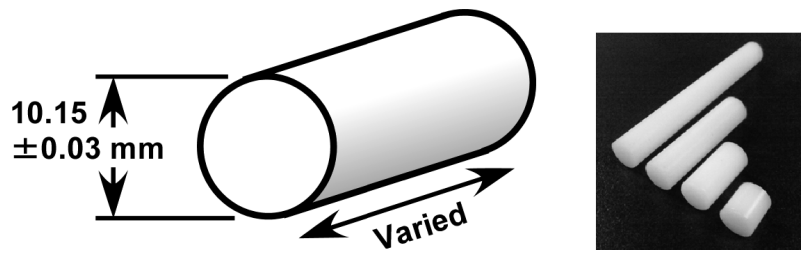

Fig. 2 Schematic and picture of projectiles.

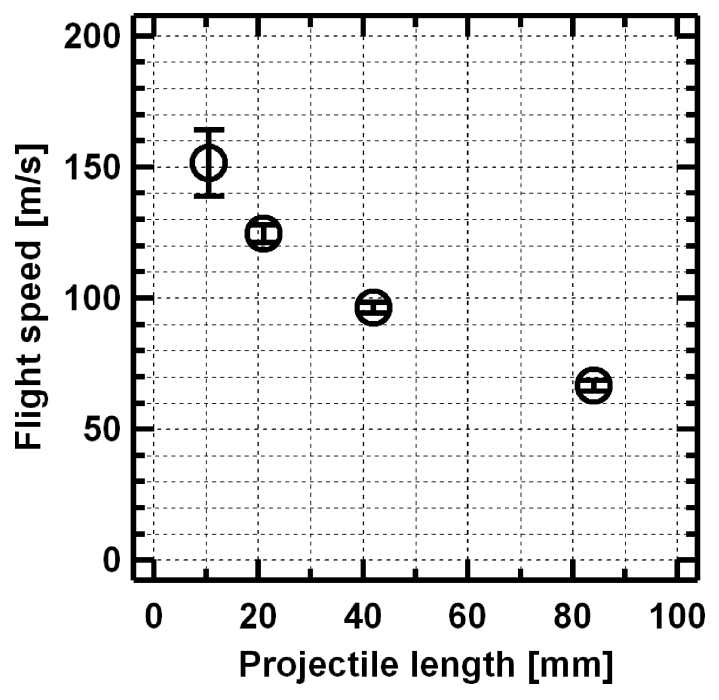

Fig. 3 Flight speed.

formula $\Delta t_{\mathrm{acc}}=2 L / V$, where $L$ and $V$ denote the length of the acceleration tube and injection speed respectively, assuming a constant acceleration. From the results of preliminary experiments, the optimum value of $\tilde{\tau}$ was estimated to be around 0.5 , and therefore, $\tilde{\tau}$ was fixed at 0.5 in the present experiments.

Figure 3 shows the measured flight speed. The error

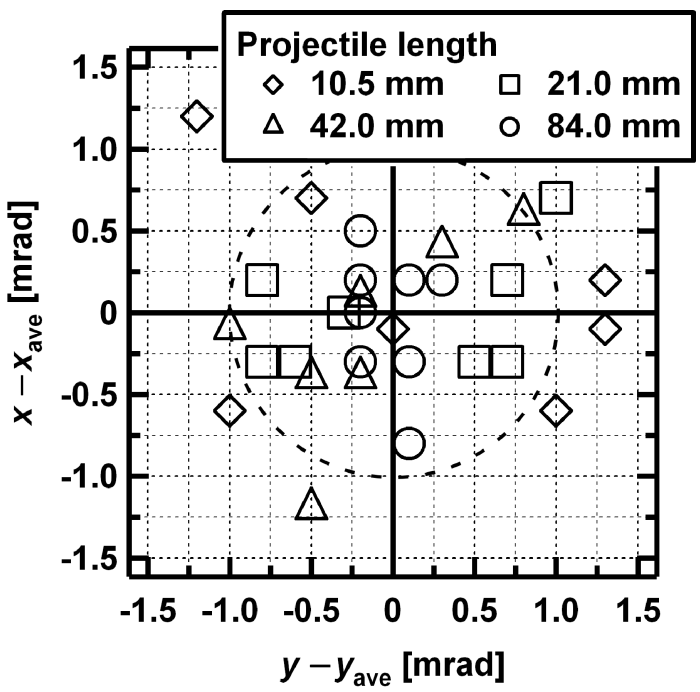

Fig. 4 Flight direction.

bars show the overall range of the scattered data. The flight speed is not considered to be critical because we can accelerate the projectiles at higher velocities by using a longer acceleration tube without raising the pressure of the accelerating gas. This will allow the projectiles to be injected with higher speed without greatly increasing disturbances due to the accelerating gas because gas distant from the exit can hardly influence the injected projectiles when the injection speed is close to the sonic speed of the gas.

The flight direction results are shown in Fig. 4. The flight direction was obtained from the position at which a projectile was stopped on the target catcher and a flight distance of $2085 \mathrm{~mm}$. The plotted flight direction is the deviation from the average for same-length projectiles. Table 1 summarizes the standard deviation of the measured flight directions. The typical required performance (one 
Table 1 Standard deviation of flight direction

\begin{tabular}{|c|c|c|}
\hline Projectile length $[\mathrm{mm}]$ & $\sigma_{x}[\mathrm{mrad}]$ & $\sigma_{y}[\mathrm{mrad}]$ \\
\hline 10.5 & 1.08 & 0.65 \\
\hline 21.0 & 0.70 & 0.35 \\
\hline 42.0 & 0.60 & 0.58 \\
\hline 84.0 & 0.18 & 0.39 \\
\hline
\end{tabular}
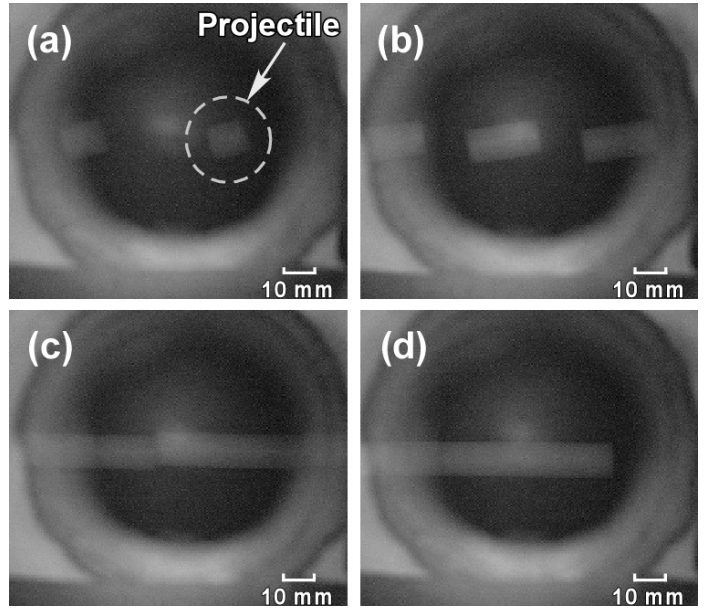

Fig. 5 Projectiles in flight. Projectile lengths are (a) $10.5 \mathrm{~mm}$, (b) $21.0 \mathrm{~mm}$, (c) $42.0 \mathrm{~mm}$, and (d) $84.0 \mathrm{~mm}$.

mrad) was attained by the $84-\mathrm{mm}$-long projectiles. In the present experiments, the resolution of the flight direction measurement was about $\pm 0.5 \mathrm{mrad}$. More precise investigation requires a more sophisticated diagnostic system.

Figure 5 shows the observed projectiles in flight. We obtained the inclination of the projectiles from these pictures. Figure 6 summarizes the results for flight attitude: the vertical axis shows the rotation speed of the projectiles, which was obtained from the observed inclination of a projectile and the flight time (flight distance divided by flight speed). The error bars show the overall range of the scattered data. As shown in Fig. 6, flight attitude was significantly improved for longer projectiles. Assuming that the flight speed is $200 \mathrm{~m} / \mathrm{s}$ and flight distance is $6 \mathrm{~m}$ in an IFE power plant, the allowable target inclination at the center of the reaction chamber of two degrees corresponds to a rotation speed of $1.2 \mathrm{rad} / \mathrm{s}$. Although the projectiles were simple cylinders in the present experiments, the 84-mm-long projectiles satisfied the requirement of flight attitude. According to the fundamental theory of dimensional analysis, it is important to determine

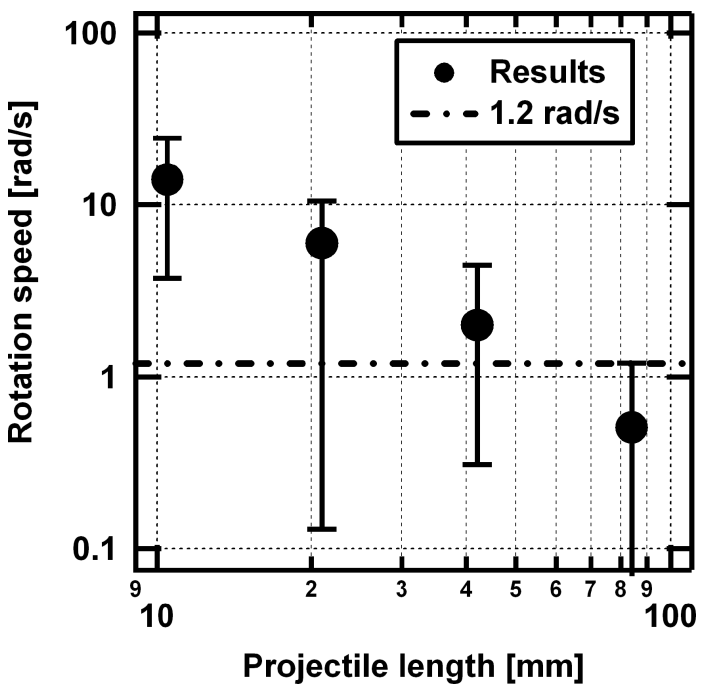

Fig. 6 Rotation speed.

the predominant governing non-dimensional parameters in designing a complicated system such as a target injection system [5]. The present experiments show that the aspect ratio of the projectile, i.e., the ratio between its length and diameter, must be a predominant non-dimensional parameter governing the performance of a target injection system. Other non-dimensional parameters such as the ratio between the projectile diameter and acceleration-tube bore diameter, where the former must be a little smaller than the latter, may be important, and studies on the effects of other parameters remain as future work.

We carried out fundamental experiments on a gas gun with smooth bore for studying key elements of a target injection system for fast-ignition-type IFE. The influence of projectile length upon injection performance was investigated in particular. Longer projectiles showed better performance. More precisely, 84-mm-long projectiles satisfied the flight direction and attitude requirements. This projectile length corresponds to approximately eight times the projectile diameter.

This work was performed under the auspices of the NIFS Collaboration Research Program (NIFS05KUGK011).

[1] T. Norimatsu et al., J. Phys. IV 133, 841 (2006).

[2] D.T. Goodin et al., Nucl. Fusion 41, 527 (2001).

[3] D.T. Goodin et al., Fusion Eng. Des. 60, 27 (2002).

[4] A.S. Bozek et al., Fusion Eng. Des. 82, 2171 (2007).

[5] M. Zlokarnik, Dimensional Analysis and Scale-up in Chemical Engineering (Springer-Verlag, Berlin, 1991). 